\title{
Localization in the Ground State of the Ising Model with a Random Transverse Field
}

\author{
Massimo Campanino ${ }^{\star}$, Abel Klein ${ }^{\star \star}$ and J. Fernando Perez ${ }^{\star \star \star}$ \\ Department of Mathematics, University of California, Irvine, Irvine, CA 92717, USA
}

Received March 21, 1990

Abstract. We study the zero-temperature behavior of the Ising model in the presence of a random transverse field. The Hamiltonian is given by

$$
H=-J \sum_{\langle x, y\rangle} \sigma_{3}(x) \sigma_{3}(y)-\sum_{x} h(x) \sigma_{1}(x),
$$

where $J>0, x, y \in \mathbf{Z}^{d}, \sigma_{1}, \sigma_{3}$ are the usual Pauli spin $\frac{1}{2}$ matrices, and $\mathbf{h}=\left\{h(x), x \in \mathbf{Z}^{d}\right\}$ are independent identically distributed random variables. We consider the ground state correlation function $\left\langle\sigma_{3}(x) \sigma_{3}(y)\right\rangle$ and prove:

1. Let $d$ be arbitrary. For any $m>0$ and $J$ sufficiently small we have, for almost every choice of the random transverse field $\mathbf{h}$ and every $x \in \mathbf{Z}^{d}$, that

$$
\left\langle\sigma_{3}(x) \sigma_{3}(y)\right\rangle \leqq C_{x, \mathbf{h}} e^{-m|x-y|}
$$

for all $y \in \mathbf{Z}^{d}$ with $C_{x, \mathbf{h}}<\infty$.

2 . Let $d \geqq 2$. If $J$ is sufficiently large, then, for almost every choice of the random transverse field $\mathbf{h}$, the model exhibits long range order, i.e.,

$$
\varlimsup_{|y| \mapsto \infty}\left\langle\sigma_{3}(x) \sigma_{3}(y)\right\rangle>0
$$

for any $x \in \mathbf{Z}^{d}$.

\section{Introduction}

Quantum spin systems with random parameters have been introduced to study the effects of impurities in several physical systems (see for example, Halperin, Lee

\footnotetext{
* Permanent address: Dipartimento di Matematica, Universita di Bologna, p.zz.a S. Donato, 5, I-40126 Bologna, Italy

$\star \star$ Partially supported by the NSF under grant DMS 8905627 and INT 8703059

$\star \star \star$ Permanent address: Instituto de Física, Universidade de São Paulo, P.O. Box 20516, CEP 01498 São Paulo, Brazil. Partially supported by the $\mathrm{CNP}_{q}$ and FAPESP
} 
and $\mathrm{Ma}$ [1] where models related to superfluidity and superconductivity were discussed).

Examples of such systems are given by quantum $X-Y$, Heisenberg and Ising models in the presence of a random transverse field. In [1] it was argued that for such models localization should take place in the ground state of the system destroying the long-range order of the non-random component of the spin system, for sufficiently high disorder.

Klein and Perez [2] have studied the quantum $X-Y$ model with a random transverse field in one dimension and proved localization in the ground state of the system for any disorder. In particular they proved exponential decay for the two-point function, which is to be compared with the polynomial decay obtained by Lieb, Schultz and Mattis [3] for zero transverse field. Their method was to map the model into a free Fermi gas in the presence of a random external potential; the one-particle Hamiltonian for the Fermi gas turned out to be the onedimensional Anderson Hamiltonian and exponential decay for the two-point function followed from Anderson localization.

In this article we study the Ising model in the presence of a random transverse field. The corresponding deterministic model appears in the pseudospin formulation of several phase transition problems and was used to study order disorder ferroelectrics with a tunneling effect by de Gennes [4] and magnetic ordering in materials with singlet crystal field ground state by Wang and Cooper [5]. The one-dimensional deterministic model was studied by Pfeuty [6] following Lieb, Schultz and Mattis [3].

The Ising model with a random transverse field is given, in a finite volume $\Lambda \subset \mathbf{Z}^{d}$, by the Hamiltonain

$$
H_{\Lambda}=-J \sum_{\langle x, y\rangle \subset \Lambda} \sigma_{3}(x) \sigma_{3}(y)-\sum_{x \in \Lambda} h(x) \sigma_{1}(x)
$$

acting on the Hilbert space $\mathscr{H}_{\Lambda}=\bigotimes_{x \in \Lambda} \mathscr{H}_{x}$, with $\mathscr{H}_{x}=\mathbf{C}^{2}$ for all $x$, where $J>0$, $\langle x, y\rangle$ denote a pair of nearest neighbor sites, $\sigma_{1}, \sigma_{3}$ are the usual Pauli spin $\frac{1}{2}$ matrices:

$$
\sigma_{3}=\left(\begin{array}{cc}
1 & 0 \\
0 & -1
\end{array}\right), \quad \sigma_{1}=\left(\begin{array}{ll}
0 & 1 \\
1 & 0
\end{array}\right)
$$

with $\sigma_{i}(x), i=1,3, x \in \Lambda$, the corresponding operator on $\mathscr{H}_{\Lambda}$ acting only on $\mathscr{H}_{x}$. The random transverse field is $\mathbf{h}=\left\{h(x), x \in \mathbf{Z}^{d}\right\}$, where the $h(x), x \in \mathbf{Z}^{d}$ are taken to be independent identically distributed random variables. Since for any $x_{0} \in \Lambda$ we have

$$
\sigma_{3}\left(x_{0}\right) H_{\Lambda} \sigma_{3}\left(x_{0}\right)=H_{\Lambda}+2 h\left(x_{0}\right) \sigma_{1}\left(x_{0}\right),
$$

we can take $h(x) \geqq 0$ without loss of generality.

If $h(x)>0$ for all $x \in \mathbf{Z}^{d}, H_{\Lambda}$ has a unique ground state $\Omega_{\Lambda}$ for each $\Lambda$ and, the correlation functions

$$
\left\langle\sigma_{3}(x) \sigma_{3}(y)\right\rangle_{\Lambda} \equiv\left(\Omega_{\Lambda}, \sigma_{3}(x) \sigma_{3}(y) \Omega_{\Lambda}\right)
$$

are monotone increasing in $\Lambda$ and decreasing functions of each $h(x)$. These follow from the representation of $H_{\Lambda}$ as the generator of a positivity improving semigroup plus correlation inequalities derived in the corresponding path space (see the 
discussion in Sect. 2). We can thus define the infinite volume ground state correlation function by

$$
\left\langle\sigma_{3}(x) \sigma_{3}(y)\right\rangle=\lim _{\Lambda \rtimes \mathbf{Z}^{d}}\left\langle\sigma_{3}(x) \sigma_{3}(y)\right\rangle_{\Lambda},
$$

preserving the monotonicity in each $h(x)$. We will always assume $\mathbf{P}\{h(x)>0\}=1$.

The deterministic uniform model, i.e., $h(x) \equiv h>0$ for all $x$, is one of the simplest quantum spin system with a non-trivial phase diagram, typical for a large class of models exhibiting discrete symmetry breakdown. The relevant parameter is $\zeta=h / J$. In any dimension there exists $0<\zeta_{1} \leqq \zeta_{2}<\infty$ such that if $\zeta>\zeta_{2}$ the correlation function (1.1) decays exponentially and if $\zeta<\zeta_{1}$ there is long range order [7,8]. In one dimension it is known that $\zeta_{1}=\zeta_{2}=2$ [8].

It follows from the monotonicity of (1.1) in each $h(x)$ that, with $\zeta(x)=h(x) / J$, the random model correlation function decays exponentially if $\zeta(x)>\zeta_{2}$ with probability one, and exhibits long range order if $\zeta(x)<\zeta_{1}$ with probability one. Thus, if $0<a \leqq h(x) \leqq b<\infty$ with probability one, the random model will exhibit a phase transition by varying $J$.

The interesting nontrivial case is thus when the events $\left\{\zeta(x)>\zeta_{2}\right\}$ and $\left\{\zeta(x)<\zeta_{1}\right\}$ both have nonzero probability, so the system exhibits Griffiths' singularities. Typical cases would be when each $h(x)$ is uniformly distributed or the interval $[0,1]$ or exponentially distributed. Let $p_{1}=\mathbf{P}\left\{\zeta(x)<\zeta_{1}\right), p_{2}=\mathbf{P}\left\{\zeta(x)>\zeta_{2}\right)$, and recall $\mathbf{P}\{h(x)>0\}=1$. Then, we have $\lim _{J \downarrow 0} p_{1}=0, \lim _{J \downarrow 0} p_{2}=1$ so for $J$ sufficiently small we should expect exponential decay of the correlation function (1.1) with probability one. On the other hand, $\lim _{J \rightarrow \infty} p_{1}=1, \lim _{J \rightarrow \infty} p_{2}=0$, so for sufficiently large the system should exhibit long range order.

Our results are

Theorem 1.1. Suppose $\mathbf{E}\left(h(x)^{-\delta}\right)<\infty$ for some $\delta>0$. Then for any $d=1,2, \ldots$ and $m>0$ there exists $J_{1}>0$ such that for any $J<J_{1}$ and for almost every choice of the random transverse field $\mathbf{h}$ and every $x \in \mathbf{Z}^{d}$ we have

$$
\left\langle\sigma_{3}(x) \sigma_{3}(y)\right\rangle \leqq C_{x, \mathbf{h}} e^{-m|x-y|}
$$

for all $y \in \mathbf{Z}^{d}$ with $C_{x, \mathbf{h}}<\infty$.

Theorem 1.2. Let $h(x)$ have an arbitrary distribution. Then for any $d \geqq 2$ there exists $J_{2}<\infty$ such that for all $J>J_{2}$ we have, for almost every choice of the random transverse field,

$$
\varlimsup_{|y| \rightarrow \infty}\left\langle\sigma_{3}(x) \sigma_{3}(y)\right\rangle>0
$$

for any $x \in \mathbf{Z}^{d}$.

Following Driessler, Landau and Perez [8] we write the correlation function (1.1) as the limit of two-point functions of $(d+1)$-dimensional classical Ising models with $d$-dimensional disorder. In Sect. 2 we show that

$$
\left\langle\sigma_{3}(x) \sigma_{3}(y)\right\rangle=\lim _{n \rightarrow \infty}\langle\sigma(x, 0) \sigma(y, 0)\rangle^{(n)},
$$

where \langle\rangle$^{(n)}$ is the expectation for the classical Ising model on $\mathbf{Z}^{d} \times \frac{1}{n} \mathbf{Z}$ with 
Hamiltonian

$$
H^{(n)}=\frac{-J}{n} \sum_{t} \sum_{\langle x, y\rangle} \sigma(x, t) \sigma(y, t)-\sum_{x} \sum_{t} K_{n}(x) \sigma(x, t) \sigma\left(x, t+\frac{1}{n}\right),
$$

where $\tanh K_{n}(x)=e^{-(2 / n) h(x)}$. Models of this type were studied by Campanino and Klein [9], who developed a multiscale expansion to prove exponential decay for the $(d+1)$-dimensional system with $d$-dimensional disorder, and gave a simple percolation argument to show long range order when $d \geqq 2$. They proved analogous results to Theorems 1.1 and 1.2 for such models, but their results can be applied directly only for $n$ fixed. In this article we refine their methods to obtain estimates uniform in $n$ for $n$ large.

This paper is organized as follows. In Sect. 2 we discuss some general features of the deterministic model, construct the associated path space and the approximation by classical Ising models. In Sect. 3 we obtain mean field type bounds on the deterministic system which will give the initial step for the multiscale analysis. Section 4 contains the multiscale analysis and the proof of Theorem 1.1. In Sect. 5 we prove Theorem 1.2.

\section{The Approximation by Classical Ising Models}

Let $\mathscr{G}_{\Lambda}=\{1,-1\}^{\Lambda}$, if $\sigma \in \mathscr{G}_{\Lambda}$ we have $\sigma=\{\sigma(x), x \in \Lambda\}$ with each $\sigma(x) \in\{1,-1\}$.

If we identify $\mathbf{C}^{2}$ with $l^{2}(\{1,-1\})$ in the obvious way we can identify $\mathscr{H}$ with $l^{2}\left(\mathscr{G}_{\lambda}\right)$; notice that the matrices of linear operators with respect to the standard base in either $\mathbf{C}^{2}$ or $\mathscr{H}_{\lambda}$ are now the kernels of the same operators on $l^{2}(\{1,-1\})$ or $l^{2}\left(\mathscr{G}_{A}\right)$, respectively.

In this representation the operator $\sigma_{3}(x)$ is given by multiplication by the function $\sigma(x)$, for each $x \in \Lambda$. Thus, if we write

with

$$
H_{\Lambda}=H_{\Lambda}^{I}+H_{\Lambda}^{t}
$$

$$
H_{\Lambda}^{I}=-J \sum_{\langle x, y\rangle \subset \Lambda} \sigma_{3}(x) \sigma_{3}(y)
$$

$H_{\Lambda}^{I}$ is given by multiplication by the function $-J \sum_{\langle x, y\rangle \subset \Lambda} \sigma(x) \sigma(y)$ and $H_{\Lambda}^{t}=$ $-\sum_{x \in \Lambda} h(x) \sigma_{1}(x)$ generate a positivity improving semigroup since $h(x)>0$ for all $x$ and

$$
e^{t \sigma_{1}}=\cosh t+(\sinh t) \sigma_{1}
$$

has a strictly positive kernel for $t>0$.

It follows from the general theory that $H_{A}$ generates a positivity improving semigroup and hence $H_{\Lambda}$ has a unique ground state $\Omega_{\Lambda}$ which is a strictly positive function. In particular, there exists a path space, i.e., a stochastic process $\{\sigma(x, t) ; x \in \Lambda, t \in \mathbf{R}\}$ taking value on $\{1,-1\}$, stationary and symmetric with respect to $t$, such that, for example,

$$
\frac{\left(\Omega_{\Lambda}, \sigma_{3}(x) e^{-|t| H_{\Lambda}} \sigma_{3}(y) \Omega_{\Lambda}\right)}{\left(\Omega_{\Lambda}, e^{-|t| H_{\Lambda}} \Omega_{\Lambda}\right)}=\langle\sigma(x, 0) \sigma(y, t)\rangle
$$


where $\langle>$ denotes the expectation in the stochastic process (see, for instance, Klein and Landau [10] for a general discussion).

In our situation $\mathscr{H}_{\Lambda}$ is finite dimensional, $H_{\Lambda}, H_{\Lambda}^{I}, H_{\Lambda}^{t}$ are therefore bounded self-adjoint operators, so it is possible to do everything more explicitly. For example, to show uniqueness of the ground, let $\lambda<H_{A}^{I}$, we have

$$
\left(H_{\Lambda}-\lambda\right)^{-1}=\left(H_{\Lambda}^{I}-\lambda\right)^{-1} \sum_{n=0}^{\infty}\left(-H_{\Lambda}^{t}\left(H_{\Lambda}^{I}-\lambda\right)^{-1}\right)^{n}
$$

the series being uniformly convergent for $\lambda \ll H_{\Lambda}^{I}$. It clearly follows that for such $\lambda,\left(H_{\Lambda}-\Lambda\right)^{-1}$ has strictly positive kernel and hence the Perron-Frobenius theorem applies so we can conclude that $H_{\Lambda}$ has a unique ground state $\Omega_{\Lambda}$ which is a strictly positive function.

The operator $H_{\Lambda}^{t}$ has the (normalized) unique ground state $\Omega_{\Lambda}^{(0)}$ given by

$$
\Omega_{\Lambda}^{(0)}(\sigma)=\frac{1}{2^{|\Lambda|}}
$$

for all $\sigma \in \mathscr{G}_{\Lambda}$. It follows immediately that

$$
\left(\Omega_{\Lambda}, \Omega_{\Lambda}^{(0)}\right)>0,
$$

so for any operator $A$ in $\mathscr{H}_{A}$ we have

$$
\langle A\rangle_{\Lambda} \equiv\left(\Omega_{\Lambda}, A \Omega_{\Lambda}\right)=\lim _{\beta \rightarrow \infty} \frac{\left(\Omega_{\Lambda}^{(0)}, e^{-(\beta / 2) H_{\Lambda}} A e^{-(\beta / 2) H_{\Lambda}} \Omega_{\Lambda}^{(0)}\right)}{\left(\Omega_{\Lambda}^{(0)}, e^{-\beta H_{\Lambda}} \Omega_{\Lambda}^{(0)}\right)} .
$$

Following Driessler, Landau and Perez [8] we can use Trotter's product formula to conclude that, if $B \subset \Lambda$,

$$
\left\langle\prod_{x \in B} \sigma_{3}(x)\right\rangle_{\Lambda} \equiv\left(\Omega_{\Lambda}, \prod_{x \in B} \sigma_{3}(x) \Omega_{\Lambda}\right)=\lim _{\beta \rightarrow \infty} \lim _{n \rightarrow \infty}\left\langle\prod_{x \in B} \sigma(x, 0)\right\rangle_{\Lambda, \beta}^{(n)}
$$

where \langle\rangle$_{\Lambda, \beta}^{(n)}$ is the expectation for the classical Ising model on $\mathbf{Z}^{d} \times \frac{1}{n} \mathbf{Z}$ with Hamiltonian given by (1.2) restricted to the region $\Lambda \times\left(\left[-\frac{\beta}{2}, \frac{\beta}{2}\right] \cap \frac{1}{n} \mathbf{Z}\right)$, with
free boundary conditions.

Since our classical Ising models are ferromagnetic and we are using free boundary conditions, we can apply correlation inequalities to obtain

$$
\left\langle\prod_{X \in \mathbf{W}} \sigma(X)\right\rangle_{\Lambda, \beta}^{(n)} \leqq\left\langle\prod_{X \in \mathbf{W}} \sigma(X)\right\rangle_{\Lambda^{\prime}, \beta^{\prime}}^{(n)}
$$

for any $\Lambda \subset \Lambda^{\prime}, \beta \leqslant \beta^{\prime}, \mathbf{W} \subset \Lambda \times\left(\left[-\frac{\beta}{2}, \frac{\beta}{2}\right] \cap \frac{1}{n} \mathbf{Z}\right)$.

Thus we can interchange the limits in $(2.1)$ to conclude

$$
\left\langle\prod_{x \in B} \sigma_{3}(x)\right\rangle_{\Lambda}=\lim _{n \rightarrow \infty}\left\langle\prod_{x \in B} \sigma(x, 0)\right\rangle_{\Lambda}^{(n)},
$$

so using again (2.2) we obtain the existence of the limit

$$
\left\langle\prod_{x \in B} \sigma_{3}(x)\right\rangle \equiv \lim _{\Lambda \rightarrow \mathbf{Z}^{d}}\left\langle\prod_{x \in B} \sigma_{3}(x)\right\rangle_{\Lambda}=\lim _{n \rightarrow \infty}\left\langle\prod_{x \in B} \sigma(x, 0)\right\rangle^{(n)} .
$$


In particular we obtain (1.1).

More generally, for a fixed finite $\Lambda$ we define

$$
\sigma_{3}(x, t)=e^{-t H_{\Lambda}} \sigma_{3}(x) e^{t H_{\Lambda}}
$$

so we have existence of

and

$$
\left\langle\sigma_{3}(x, t) \sigma_{3}(y, s)\right\rangle \equiv \lim _{\Lambda \rightarrow \mathbf{Z}^{d}}\left\langle\sigma_{3}(x, t) \sigma_{3}(y, s)\right\rangle_{\Lambda}
$$

$$
\left\langle\sigma_{3}(x, t) \sigma_{3}(y, s)\right\rangle=\lim _{n \rightarrow \infty}\left\langle\sigma\left(x, t^{(n)}\right) \sigma\left(y, s^{(n)}\right)\right\rangle^{(n)},
$$

where for $r \in \mathbf{R}$ we let $r^{(n)}=\frac{1}{n}[n r]$ if $r \geqq 0$ and $r^{(n)}=-|r|^{(n)}$ if $r<0$.

\section{Percolation, Self-Avoiding Walks and Mean-Field Bounds}

For each $n$ we consider the bond Bernoulli percolation model on $\mathbf{Z}^{d} \times \frac{1}{n} \mathbf{Z}$ with occupation probabilities

$$
q_{(x, t),(y, s)}^{(n)}= \begin{cases}1-e^{-2(J / n)} & \text { if } t=s, x, y \text { nearest neighbors } \\ 1-e^{-2 K_{n}(x)} & \text { if } x=y,|t-s|=\frac{1}{n} \\ 0 & \text { otherwise }\end{cases}
$$

The corresponding percolation probability will be denoted by $\mathbf{Q}^{(n)}$; notice that it depends on the choice of the random transverse field $\mathbf{h}$.

If $\mathbf{W} \subset \mathbf{Z}^{d} \times \mathbf{R}$, we set $\mathbf{W}^{(n)}=\mathbf{W} \cap\left(\mathbf{Z}^{d} \times \frac{1}{n} \mathbf{Z}\right)$. If $X, Y \in \mathbf{W}^{(n)}$, by $X \underset{\mathbf{w}}{\rightarrow} Y$ we mean that $X$ is connected to $Y$ by a path of occupied bonds in $\mathbf{W}^{(n)}$. We set

$$
G_{\mathbf{W}}^{(n)}(X, Y)=\mathbf{Q}^{(n)}\{X \underset{\mathbf{w}}{\rightarrow} Y\}
$$

It follows from the Fortuin-Kasteleyn representation of Ising models and from Fortuin's comparison principles (see Aizenman et al. [11]) that

$$
\langle\sigma(X) \sigma(Y)\rangle_{\mathrm{w}}^{(n)} \leqq G_{\mathrm{w}}^{(n)}(X, Y),
$$

where the left-hand-side denotes the two-point of the classical Ising model in $\mathbf{Z}^{d} \times \frac{1}{n} \mathbf{Z}$ with Hamiltonian given by (1.2), restricted to the region $\mathbf{W}^{(n)}$ with free boundary condition.

Following Campanino and Klein [9], we will prove Theorem 1.1 by showing decay for $G^{(n)}(X, Y)$.

Since if $X \underset{\mathbf{w}}{\longrightarrow} Y$ we can always find a self-avoiding walk in $\mathbf{W}^{(n)}$ starting at $X$ and ending at $Y$, we also have

where

$$
G_{\mathrm{w}}^{(n)}(X, Y) \leqq S_{\mathrm{w}}^{(n)}(X, Y)
$$

$$
S_{W}^{(n)}(X, Y)=\sum_{w} q_{w}^{(n)},
$$


the summation being taken over all nearest neighbors self-avoiding walks $w$ in $\mathbf{W}^{(n)}$ that go from $X$ to $Y$, i.e., $W:\{0,1, \ldots,|w|\} \rightarrow \mathbf{W}^{(n)}$ with $w(0)=X, w(|w|)=Y$, and $w(i), w(i+1)$ nearest neighbors, $w(i) \neq w(j)$ if $i \neq j$. Here

$$
q_{W}^{(n)}=\prod_{i=0}^{|w|-1} q_{w(i), w(i+1)}^{(n)} .
$$

Another similar bound for $\langle\sigma(X) \sigma(Y)\rangle^{(n)}$ (but not for $G^{(n)}(X, Y)$ ) was obtained by Fisher [12]:

$$
\langle\sigma(X) \sigma(Y)\rangle_{\mathrm{w}}^{(n)} \leqq \widetilde{S}_{\mathrm{w}}^{(n)}(X, Y),
$$

where $\widetilde{S}_{\mathbf{W}}^{(n)}$ is defined also by (3.3) but with $q_{x, y}^{(n)}$ replaced by

$$
\tilde{q}_{(x, t),(y, s)}^{(n)}=\left\{\begin{array}{ll}
\tanh \frac{J}{n} & \text { if } t=s, x, y \text { nearest neighbors } \\
\tanh K_{n}(x) & \text { if } x=y,|t-s|=\frac{1}{n} \\
0 & \text { otherwise }
\end{array} .\right.
$$

Let $\mathbf{W}=\Lambda \times[-T, T]$, with $\Lambda \subset \mathbf{Z}^{d}$. Following Fisher [12] we estimate (3.3) by replacing the self-avoiding condition by the weaker requirement of no immediate return after a vertical step, obtaining

$$
S_{\Lambda \pm[-T, T]}^{(n)}((x, t),(y, s)) \leqq \sum_{J: x \rightarrow y} \sum_{k_{0}, \ldots, k_{|\tau|}}^{\prime} \prod_{i=0}^{|\tau|}\left(1-e^{-2 K_{n}(\tau(i))}\right)^{\left|k_{\imath}\right|}\left(1-e^{-2(J / n)}\right)^{|\tau|}
$$

where the first summation runs over all walks $\tau$ in $\Lambda$ from $x$ to $y$, the second summation being over the number of vertical steps $k_{i}$ taken by $\tau$ after the $i^{\text {th }}$ horizontal step, with $k_{i}>0$ if the steps are upwards, $k_{i}<0$ if downwards, the prime in the summation accounts for the restriction

$$
\sum_{i=0}^{|\tau|} k_{i}=(s-t)^{n}
$$

In particular,

$$
\sum_{i=0}^{|\tau|}\left|k_{i}\right| \geqq|s-t| n
$$

Recall $e^{-2 K_{n}(x)}=\tanh \frac{h(x)}{n}$, and set $h_{\Lambda}=\min _{x \in \Lambda} h(x)$. We get

$$
\begin{aligned}
& S_{\Lambda \times[-T, T]}^{(n)}((x, t),(y, s)) \\
& \leqq \sum_{\tau: x \rightarrow y}\left(1-e^{-(2 J / n)}\right)^{|\tau|} \sum_{k_{0}, k_{1}, \ldots, k_{|\tau|}}^{\prime}\left(1-\tanh \frac{h_{\Lambda}}{n}\right)^{|\tau|} \sum_{i=0}^{\left|k_{i}\right|} \\
& \leqq\left(1-\tanh \frac{h_{A}}{n}\right)^{\delta n|t-s|} \sum_{\tau: x \rightarrow y}\left(1-e^{-(2 J / n)}\right)^{|\tau|} \sum_{k_{0}, k_{1}, \ldots, k_{|\tau|}}^{\prime}\left(1-\tanh \frac{h_{\Lambda}}{n}\right)^{(1-\delta)} \sum_{l=0}^{|\tau|} k_{i} \mid \\
& \leqq\left(1-\tanh \frac{h_{\Lambda}}{n}\right)^{\delta n|t-s|} \sum_{\tau: x \rightarrow y} \sum_{k_{0}, k_{1}, \ldots, k_{|\tau|-1}}^{\prime}\left(1-\tanh \frac{h_{\Lambda}}{n}\right)^{(1-\delta)} \sum_{i=0}^{|\tau|-1}\left|k_{i}\right|
\end{aligned}
$$


for any $0 \leqq \delta<1$, using (3.7) and (3.6); where the sum over $k_{0}, \ldots, k_{|\tau|-1}$ is now unrestricted.

Thus, we have

$$
S_{\Lambda \times[-T, T]}^{(n)}((x, t),(y, s)) \leqq\left(1-\tanh \frac{h_{\Lambda}}{n}\right)^{\delta n|t-s|} \sum_{\tau: x \rightarrow y} \zeta_{n, \delta}^{|\tau|}
$$

where

$$
\zeta_{n, \delta}=\frac{2\left(1-e^{-2(J / n)}\right)}{1-\left(1-\tanh \frac{h}{n}\right)^{1-\delta}}
$$

If $0<2 d R<1$, we have

$$
\sum_{\tau: x \rightarrow y} R^{|\tau|}=\left(-R \Delta_{\Lambda}+1\right)^{-1}(x, y) \leqq \frac{(2 d R)^{|x-y|}}{1-2 d R},
$$

where $\Delta_{\Lambda}$ is the centered Laplacian in $\Lambda \subset \mathbf{Z}^{d}$, i.e., $\Delta_{\Lambda}(x, y)=1$ if $x$ and $y$ in $\Lambda$ are nearest neighbors, and equals zero otherwise. Thus it follows from (3.8) and (3.9) that

$$
\begin{aligned}
\left.S_{\Lambda \times[-T, T]}^{(n)}((x, t),(y, s))\right) & \leqq\left(1-\tanh \frac{h_{\Lambda}}{n}\right)^{\delta n|t-s|}\left(-\zeta_{n, \delta} \Delta_{\Lambda}+1\right)^{-1}(x, y) \\
& \leqq\left(1-\tanh \frac{h_{\Lambda}}{n}\right)^{\delta n|t-s|} \frac{\left(2 d \zeta_{n, \delta}\right)^{|x-y|}}{1-2 d \zeta_{n, \delta}} .
\end{aligned}
$$

We have

$$
\lim _{n \rightarrow \infty} \zeta_{n, \delta}=\frac{4 J}{(1-\delta) h_{\Lambda}}
$$

It follows that, if $\frac{8 d J}{(1-\delta) h_{\Lambda}}<1$, we have

$$
\begin{aligned}
\varlimsup_{n \rightarrow \infty} S_{\Lambda \times[-T, T]}^{(n)}\left(\left(x, t^{(n)}\right),\left(y, s^{(n)}\right)\right) & \leqq e^{-\delta h_{\Lambda}|t-s|}\left(-\frac{4 J}{(1-\delta) h_{\Lambda}} \Delta_{\Lambda}+1\right)^{-1}(x, y) \\
& \leqq\left(1-\frac{8 d J}{(1-\delta) h_{\Lambda}}\right)^{-1} e^{-\delta h_{\Lambda}|t-s|}\left(\frac{8 d J}{(1-\delta) h_{\Lambda}}\right)^{|x-y|}
\end{aligned}
$$

for any $0<\delta<1$.

Similarly, if $\frac{2 d J}{(1-\delta) h_{\Lambda}}<1$, we have

$$
\begin{aligned}
\varlimsup_{n \rightarrow \infty} \tilde{S}_{\Lambda \times[-T, T]}^{(n)}\left(\left(x, t^{(n)}\right),\left(y, s^{(n)}\right)\right) & \leqq e^{-2 \delta h_{\Lambda}|t-s|}\left(-\frac{J}{(1-\delta) h_{\Lambda}} \Delta_{\Lambda}+1\right)^{-1}(x, y) \\
& \leqq\left(1-\frac{2 d J}{(1-\delta) h_{\Lambda}}\right)^{-1} e^{-2 \delta h_{\Lambda}|t-s|}\left(\frac{2 d J}{(1-\delta) h_{\Lambda}}\right)^{|x-y|}
\end{aligned}
$$


In particular, from (1.1), (2.3), (3.4) and (3.12) we immediately get

Theorem 3.1. Suppose $\bar{h}=\inf _{x \in \mathbf{Z}^{d}} h(x)>0$.

Then, if $2 d J<\bar{h}$ we have

$$
\left\langle\sigma_{3}(x) \sigma_{3}(y)\right\rangle \leqq\left(-\frac{J}{\bar{h}} \Delta+1\right)^{-1}(x, y) \leqq\left(1-\frac{2 d J}{\bar{h}}\right)^{-1}\left(\frac{2 d J}{\bar{h}}\right)^{|x-y|}
$$

for all $x, y \in \mathbf{Z}^{d}$.

More generally, we have

$$
\begin{aligned}
\left\langle\sigma_{3}(x, t) \sigma_{3}(y, s)\right\rangle & \leqq e^{-2 \delta \bar{h}|t-s|}\left(\frac{-J}{(1-\delta) \bar{h}} \Delta+1\right)^{-1}(x, y) \\
& \leqq\left(1-\frac{2 d J}{(1-\delta) \bar{h}}\right)^{-1} e^{-2 \delta \bar{h}|t-s|}\left(\frac{2 d J}{(1-\delta) \bar{h}}\right)^{|t-y|}
\end{aligned}
$$

for any $x, y \in \mathbf{Z}^{d}, t, s \in \mathbf{R}$ and all $0 \leqq \delta<1$ such that $2 d J<(1-\delta) \bar{h}$.

\section{The Multiscale Analysis}

We will now prove Theorem 1.1. Our proof follows the proof of Theorem 2.1 in [9], the main difference is that we need to control the limit as $n \rightarrow \infty$ in (1.1) so we must perform a multiscale analysis uniformly in $n$ for $n$ large.

In view of (3.1) Theorem 1.1 will follows from

Theorem 4.1. Suppose $\mathbf{E}\left(h(x)^{-\delta}\right)<\infty$ for some $\delta>0$. Then for any $d \geqq 1, m>0$ and $v>1$ there exists $J_{1}>0$ and $n_{1}<\infty$ such that if $J<J_{1}$, then for almost every choice of $\mathbf{h}$ we have

$$
G^{(n)}((x, t),(y, s)) \leqq C_{x, \mathbf{h}} e^{m\left|\left(x-y,(\log |t-s|)^{v}\right)\right|}
$$

for all $n \geqq n_{1}, x, y \in \mathbf{Z}^{d}, t, s \in \mathbf{R}$, with $C_{x, \mathbf{h}}=C_{x, \mathbf{h}}(J, m, v)<\infty$.

We will restrict ourselves to the case $v=2$, the modification for arbitrary $v>1$ will be clear. Notice we use the notation $|(x, t)|=\max \{|x|,|t|\}$, where $|x| \equiv\|x\|_{\infty}$ for $x \in \mathbf{Z}^{d}$.

The proof of Theorem 4.1 will use properties of independent bond percolation, including the Harris-FKG, van der Berg-Kesten (v-BK) and HammersleySimon-Lieb (HSL) inequalities. The first two will be used as described in [9], but we will need a slightly different form of the HSL inequality which follows from the $\mathrm{v}-\mathrm{BK}$ inequality. Let $\Lambda, \Lambda^{\prime} \subset \mathbf{Z}^{d}, I, I^{\prime} \subset \mathbf{R}, \mathbf{W}=\Lambda \times I, \mathbf{W}^{\prime}=\Lambda^{\prime} \times I^{\prime}$. Let $\mathbf{W}^{(n)}=\mathbf{W} \cap\left(\mathbf{Z}^{d} \times \frac{1}{n} \mathbf{Z}\right)$,
similarly for $\mathbf{W}^{\prime(n)}$. We set

$$
\begin{aligned}
& \partial_{H}^{(n)}\left(\mathbf{W}, \mathbf{W}^{\prime}\right)=\left\{(y, s) \in \mathbf{W}^{(n)} \cap \mathbf{W}^{\prime(n)} \text {; where }\left(y, s+\frac{1}{n}\right) \text { or }\left(y, s-\frac{1}{n}\right) \in \mathbf{W}^{\prime(n)} \backslash \mathbf{W}^{(n)}\right\}, \\
& \partial_{V}^{(n)}\left(\mathbf{W}, \mathbf{W}^{\prime}\right)=\left\{\left\langle(y, s),\left(y^{\prime}, s\right)\right\rangle ;(y, s) \in \mathbf{W}^{(n)} \cap \mathbf{W}^{\prime(n)},\left(y, s^{\prime}\right) \in \mathbf{W}^{\prime(n)} \backslash \mathbf{W}^{\prime(n)}\right\} .
\end{aligned}
$$


If $\mathbf{W}^{\prime}=\mathbf{Z}^{d} \times \mathbf{R}$, we omit $\mathbf{W}^{\prime}$. We also write $\partial^{(n)} \mathbf{W}=\partial_{H}^{(n)} \mathbf{W} \cup\left\{Z ;\left\langle Z, Z^{\prime}\right\rangle \in \partial_{V}^{(n)} \mathbf{W}\right.$ for some $\left.Z^{\prime}\right\}$.

Now let $X \in \mathbf{W}^{(n)} \cap \mathbf{W}^{\prime(n)}, Y \in \mathbf{W}^{\prime(n)} \backslash \mathbf{W}^{(n)}$, it follows from the HSL inequality that

$$
\begin{aligned}
G_{\mathbf{W}^{\prime}}^{(n)}(X, Y) \leqq & \sum_{Z \in \partial_{H}^{(n)}\left(\mathbf{W}, \mathbf{W}^{\prime}\right)} G_{\mathbf{W} \cap \mathbf{W}^{\prime}}^{(n)}(X, Z) G_{\mathbf{W}^{\prime}}^{(n)}(Z, Y) \\
& +\left(1-e^{-2(J / n)}\right) \sum_{\left\langle Z, Z^{\prime}\right\rangle \in \partial_{V}^{(n)}\left(\mathbf{W}, \mathbf{w}^{\prime}\right)} G_{\mathbf{W} \cap \mathbf{W}^{\prime}}^{(n)}(X, Z) G_{\mathbf{W}^{\prime}}^{(n)}\left(Z^{\prime}, Y\right) .
\end{aligned}
$$

For large $n, 1-e^{-2 J / n} \approx \frac{2 J}{n} ;$ this factor is needed in (4.1) since a vertical line of length $T$ contains $n T$ points of $\mathbf{Z}^{d} \times \frac{1}{n} \mathbf{Z}$.

We will use the following consequence of (4.1). If $X \in \mathbf{W}^{(n)}$, let

$$
G_{\mathbf{W}}^{(n)}(X, \partial)=\sum_{Z \in \partial_{H}^{(n)} \mathbf{W}} G_{\mathrm{W}}^{(n)}(X, Z)+\left(1-e^{-2 J / n}\right) \sum_{\left\langle Z, Z^{\prime}\right\rangle \in \partial_{V}^{(n)} \mathbf{W}} G_{\mathrm{W}}^{(n)}(X, Z) .
$$

Then, for $X \in \mathbf{W}^{(n)} \cap \mathbf{W}^{\prime(n)}, Y \in \mathbf{W}^{\prime(n)} \backslash \mathbf{W}^{(n)}$, we have

$$
G_{\mathrm{w}^{\prime}}^{(n)}(X, Y) \leqq G_{\mathrm{W}}^{(n)}(X, \partial) G_{\mathrm{W}^{\prime}}\left(Z_{1}, Y\right)
$$

for some

$$
Z_{1} \in \partial_{H}^{(n)}\left(\mathbf{W}, \mathbf{W}^{\prime}\right) \cup\left\{Z^{\prime} ;\left\langle Z, Z^{\prime}\right\rangle \in \partial_{V}^{(n)}\left(\mathbf{W}, \mathbf{W}^{\prime}\right) \text { for some } Z\right\} .
$$

We will now start the multiscale analysis. For $x \in \mathbf{Z}^{d}$ and $L>0$ let us consider the hypercube

$$
\Lambda_{L}(x)=\left\{y \in \mathbf{Z}^{d} ;|x-y| \leqq L\right\} .
$$

For $X=(x, t) \in \mathbf{Z}^{d} \times \mathbf{R}, L>0, T>0$, we consider the cylinder

$$
B_{L, T}(X)=\Lambda_{L}(x) \times[t-T, t+T]
$$

and, in particular

$$
B_{L}(X)=B_{L, e^{\sqrt{ }}(X)}
$$

Definitions. Let $m>0, L>0, \bar{n}>0$. A site $x \in \mathbf{Z}^{d}$ is said to be $(m, L, \bar{n})$-regular if

$$
G_{B_{L}((x, 0))}^{(n)}((x, 0), Y) \leqq e^{-m L}
$$

for all $n \geqq \bar{n}$ and $Y \in \partial^{(n)} B_{L}((x, 0))$. Otherwise $x$ is said to be $(m, L, \bar{n})$-singular. A set $\Lambda \subset \mathbf{Z}^{d}$ is called $(m, L, \bar{n})$-regular if every $x \in \Lambda$ is $(m, L, \bar{n})$-regular.

If $x$ is $(m, L, \bar{n})$-regular we have

$$
G_{B_{L}((x, t))}^{(n)}((x, t), \partial) \leqq e^{-(m-2 / \sqrt{L}) L}
$$

for all $n>\bar{n}, t \in \mathbf{R}$ and all $L$ sufficiently large.

Theorem 4.2. Assume $\mathbf{E}\left(h(x)^{-\delta}\right)<\infty$ for some $\delta>0$. Fix $J>0$, and let $p>2 d^{2}$. Suppose there exists $m_{0}>0, L_{0}>0$ and $n_{0}>0$ such that

$$
\mathbf{P}\left\{0 \text { is }\left(m_{0}, L_{0}, n_{0}\right)-\text { regular }\right\} \geqq 1-\frac{1}{L_{0}^{p}} \text {. }
$$

Let $\alpha \in\left(2 d, \frac{p}{d}\right)$, set $L_{k+1}=L_{k}^{\alpha}, k=0,1, \ldots$ Then for any $0<m_{\infty}<m_{0}$ there exists 
$\bar{L}\left(p, d, m_{0}, m_{\infty}, \alpha, J\right)<\infty$, nondecreasing in $J$, such that if $L_{0}>\bar{L}$ there exists $\bar{n}<\infty$ such that

$$
\mathbf{P}\left\{0 \text { is }\left(m_{\infty}, L_{k}, \bar{n}\right)-\text { regular }\right\} \geqq 1-\frac{1}{L_{k}^{p}}
$$

for all $k=0,1,2, \ldots$.

Theorem 4.2 implies Theorem 4.1. For given $m>0$ let $m_{0}=2 m, m_{2}=m$, take $\Lambda=\Lambda_{L}(0)$ and suppose that

$$
h_{\Lambda}>16 d J e^{2 m_{0}}
$$

and

$$
h_{\Lambda}>4 m_{0} L e^{\sqrt{L}} .
$$

It follows from (3.2) and (3.11) that if (4.4) and (4.5) hold we have that 0 is ( $m_{0}, L, n_{1}$ )-regular for some $n_{1}>\infty$ if $J$ is sufficiently small and $L$ sufficiently large.

Let $E_{J, L}$ be the event that (4.4) and (4.5) hold. We have

$$
\begin{aligned}
\mathbf{P}\left(E_{J, L}^{c}\right) & \leqq(2 L+1)^{d}\left[\mathbf{P}\left\{h(0) \leqq 16 d J e^{2 m_{0}}\right\}+\mathbf{P}\left\{h(0) \leqq 4 m_{0} L e^{\sqrt{L}}\right\}\right] \\
& \leqq(2 L+1)^{d} \mathbf{E}\left(h(0)^{-\delta}\right)\left[\left(16 d J e^{2 m_{0}}\right)^{\delta}+\left(4 m_{0} L e^{-\sqrt{L}}\right)^{\delta}\right] .
\end{aligned}
$$

Thus there exist $J_{\alpha}>0, \tilde{L}<\infty$ such that if $J \leqq J_{\alpha}, L>\tilde{L}$ we have

$$
\mathbf{P}\left(E_{J, L}\right) \geqq 1-\frac{1}{L^{p}} .
$$

Now pick $\bar{L}\left(J_{2}\right)$ from Theorem 4.2, take $L_{0}>\max \left\{\bar{L}\left(J_{\alpha}\right), \tilde{L}\right\}$, and pick $0<J_{1} \leqq J_{2}$ such that $\mathbf{P}\left(E_{J, L_{0}}\right) \geqq 1-\frac{1}{L_{0}^{p}}$ for all $J \leqq J_{1}$. Since $\bar{L}(J)$ is nondecreasing in $J$ we have $\bar{L}(J)<L_{0}$ and hence we can apply Theorem 4.1 for $J \leqq J_{1}$.

Theorem 4.1 now follows from Theorem 4.2 by the proof of Corollary 3.2 in [9]. Notice that under the conclusions of Theorem 4.2 the estimates can be done uniformly in $n$ for $n \geqq \bar{n}$.

Theorem 4.2 is proved in a similar way to Theorem 3.1 in [9]. Again, the main difference is that the estimates have to be done uniformly in $n$ for $n$ large enough. This has been built in our definitions of regular sites and regular regions, which include the uniformity in $n$ for all $n$ large enough.

For the benefit of the reader we will sketch the proof stating clearly the main steps in the framework of this paper and highlighting the differences from [9]. lemma.

Theorem 4.2 is proven by induction. The induction step is given by the following

Lemma 4.3. Let $p>2 d^{2}, \alpha \in(2 d, p)$ and $L=l^{\alpha}$. Suppose

$$
\mathbf{P}\{0 \text { is }(m, l, \bar{n})-\text { regular }\} \geqq 1-\frac{1}{l^{p}}
$$

with $m \geqq \frac{3}{\sqrt{l}}$. Then, we have

$$
\mathbf{P}\left\{0 \text { is }(M, L, \bar{n})-\text { regular } \geqq 1-\frac{1}{L^{p}}\right.
$$


with

$$
M \geqq m-\left(a_{1} m+a_{2}\right) \frac{1}{\sqrt{l}} \geqq \frac{3}{\sqrt{L}}
$$

for some constants $a_{1}, a_{2}$ independent of $l$, in case $l$ and $\bar{n}$ are sufficiently large.

As in the proof of Lemma 3.5 in [9], one starts by picking a positive integer $R$ such that

$$
\alpha<\frac{(R+1) p}{p+(R+1) d} .
$$

For $l$ large enough we can show that

$$
\begin{aligned}
& \mathbf{P}\left\{\text { there exists } x_{1}, \ldots, x_{R} \in \Lambda_{L}(0) \text { such that } \Lambda_{L}(0) \backslash \bigcup_{j=0}^{R} \Lambda_{2 l}\left(x_{j}\right)\right. \\
& \text { is a }(m, l, \bar{n})-\text { regular region }\} \geqq 1-\frac{1}{2 L^{p}} .
\end{aligned}
$$

We now want to estimate $G_{B_{L}(0)}^{(n)}(0, Y)$ for $Y \in \partial^{(n)} B_{L}(0)$ and $n \geqq \bar{n}$. There are two distinct cases: either $Y$ is in the vertical boundary $\partial_{V}^{(n)} B_{L}(0)$ or in the horizontal boundary $\partial_{H}^{(n)} B_{L}(0)$. We can restrict ourselves to the case when the event described in (4.6) holds.

Sublemma 4.4. Suppose there exist $x_{1}, \ldots, x_{R} \in \Lambda_{L}(0)$ such that

$$
\Lambda_{L}(0) \backslash \bigcup_{j=1}^{R} \Lambda_{2 l}\left(x_{j}\right)
$$

is $(m, l, \bar{n})$-regular region. Then, if $l$ is sufficiently large and $m>\frac{3}{\sqrt{l}}$, we have

$$
G_{B_{L}(0)}^{(n)}(0, Y) \leqq e^{-M_{1} L}
$$

with

$$
M_{1} \geqq m-\left(a_{3} m+a_{4}\right) \frac{1}{\sqrt{l}} \geqq \frac{3}{\sqrt{L}}
$$

for all $Y \in \partial_{V}^{(n)} B_{L}(0)$ and $n \geqq \bar{n}$, for some constant, $a_{3}, a_{4}$ independent of $l$ and $n$.

Proof. Same as Sublemma 3.6 in [9].

Sublemma 4.5. Suppose there exist $x_{1}, \ldots, x_{R} \in \Lambda_{L}(0)$ such that $\Lambda^{\prime}=\Lambda_{L(0)} \backslash \bigcup_{j=1}^{R} \Lambda_{l^{k}}\left(x_{j}\right)$ is a $(m, l, \bar{n})$-regular for $1<\kappa<\frac{\alpha}{2 d}$,

$$
\tilde{\Lambda}=\left(\bigcup_{j=1}^{R} \Lambda_{l^{\kappa}}\left(x_{j}\right)\right) \cap \Lambda_{L}(0)
$$

and suppose

$$
e^{2 J d|\tilde{\Lambda}|} \prod_{x \in \tilde{\Lambda}}\left(1-\left(1-\tanh \frac{h(x)}{n}\right)^{n}\right) \leqq e^{-\sigma|\tilde{\Lambda}|}
$$


for all $n \geqq \bar{n}$, where $\sigma>0$ is a given constant. Then, if $m \geqq \frac{3}{\sqrt{l}}, \frac{1}{2}<\tau<\kappa-\frac{1}{2}$, we have $G_{B_{L}(0)}^{(n)}(0, Y) \leqq e^{-M_{2} e^{t^{t} / 4}}$

for $Y \in \partial_{H}^{(n)} B_{L}(0), n \geqq \bar{n}$, with

$$
M_{2} \geqq m-e^{-l^{\tau} / 4}(m+1)
$$

for $l$ sufficiently large.

Proof. The proof proceeds as in the proof of Sublemma 3.7 in [9] with one important modification. The main difficulty in the proof is how to control the percolation inside the cylinder based on the singular region. This was done in [9] by introducing the event $D_{s}$ of the existence of a vertical disconnection at height $s$ in a certain neighborhood of the singular region. In this paper we replace $D_{s}$ by the events

$$
\begin{aligned}
& D_{s}^{(n)}=\{\text { all horizontal bonds }\langle(x, t),(y, t)\rangle \text { are vacant for } x, y \in \tilde{\Lambda} \text {, nearest } \\
& \text { neighbors, } t \in[s, s+1] \cap \frac{1}{n} \mathbf{Z} \text {, and for each } x \in \tilde{\Lambda} \text { at least one vertical bond } \\
& \text { of the type } \left.\left\langle(x, t),\left(x, t+\frac{1}{n}\right)\right\rangle, t, t+\frac{1}{n} \in[s, s+1] \cap \frac{1}{n} \mathbf{Z} \text { is vacant }\right\}
\end{aligned}
$$

We have

$$
\begin{aligned}
D_{s}^{(n)} \subset\{ & \left\{\text { there is no connection from } \tilde{\Lambda} \times\left\{s^{(n)}\right\} \text { to } \tilde{\Lambda} \times\left\{(s+1)^{(n)}\right\},\right. \\
& \text { contained in } \left.\tilde{\Lambda} \times \frac{1}{n} \mathbf{Z}\right\},
\end{aligned}
$$

and

$$
\mathbf{Q}^{(n)}\left(D_{s}^{(n)}\right) \geqq\left(e^{-2 J / n}\right)^{n d|\tilde{\Lambda}|} \prod_{x \in \tilde{\Lambda}}\left(1-\left(1-\tanh \frac{h(x)}{n}\right)^{n}\right) .
$$

By (4.7) we have

$$
\mathbf{Q}^{(n)}\left(D_{s}^{(n)}\right) \geqq e^{-\sigma\left(2 l^{\kappa}+1\right)^{d} R} \geqq e^{-\xi l^{\kappa d}},
$$

where $\xi=3^{d} \sigma R$, for all $n \geqq \bar{n}$. Apart from this modification the proof is identical to that of Sublemma 3.7 in [9].

To finish the proof of Lemma 4.3 we need only to show that

$$
\mathbf{P}\{(4.7) \text { holds for all } n \geqq \vec{n}\} \geqq 1-\frac{1}{2 L^{p}} \text {. }
$$

This follows from the following lemma.

Lemma 4.6. Let $\mu=\log \mathbf{E}\left(\left(1-e^{-h(x)}\right)^{-\delta}\right)$,

$$
\sigma=2 \delta^{-1}(2 d J \delta+\mu+\log 2), \quad v=\delta \sigma .
$$


Then, there exists $\bar{n}<\infty$ such for all $n \geqq \bar{n}$ and all $\Lambda \subset \mathbf{Z}^{d}$ finite we have

$$
\mathbf{P}\left\{e^{-2 d J|\Lambda|} \prod_{x \in \Lambda}\left(1-\left(1-\tanh \frac{h(x)}{n}\right)^{n}\right) \leqq e^{-\sigma|\Lambda|}\right\} \leqq e^{-v|\Lambda|}
$$

Proof. Notice $\mu<\infty$ since $\mathbf{E}\left(h(x)^{-\delta}\right)<\infty$. Using Chebychev's inequality we get

$$
\begin{aligned}
& \mathbf{P}\left\{e^{-2 d J|\Lambda|} \prod_{x \in \Lambda}\left(1-\left(1-\tanh \frac{h(x)^{n}}{n}\right) \leqq \varepsilon\right\}\right. \\
& \quad \leqq \varepsilon^{\delta} e^{2 d J|\Lambda|}\left[\mathbf{E}\left[\left(\left(1-\left(1-\tanh \frac{h(x)}{n}\right)^{n}\right)^{-\delta}\right]\right]^{|\Lambda|} .\right.
\end{aligned}
$$

Since $\lim _{n \rightarrow \infty}\left(1-\tanh \frac{h}{n}\right)^{n}=e^{-h}$, there exists $\bar{n}$ such that if $n \geqq \bar{n}$ we have

$$
\mathbf{E}\left[\left(\left(1-\left(1-\tanh \frac{h}{n}\right)^{n}\right)^{-\delta}\right] \leqq 2 \mathbf{E}\left[\left(1-e^{-h}\right)^{-\delta}\right] .\right.
$$

Choosing $\varepsilon=e^{-\sigma|\Lambda|}$, the result follows.

This finishes the proof of Theorem 4.2.

\section{Long Range Order}

We first discuss the existence of long-range order and spontaneous magnetization in the ground state for the uniform deterministic model. Related results may be found in the literature (see Ginibre [7] for a finite temperature discussion, Pfeuty [6] for an explicit solution in $d=1$ with periodic boundary conditions, and also Driessler, Landau and Perez [8]), but none are in the form needed in this work.

Our Peierls' argument is performed in the classical Ising model approximation coupled with Fisher's trick [12] for summing over contours, which allows estimates uniform in $n$.

Let, for $x \in \mathbf{Z}^{d}$.

$$
P_{ \pm}(x)=\frac{1 \pm \sigma_{3}(x)}{2}
$$

Then

$$
\left\langle P_{+}(x) P_{-}(y)\right\rangle=\lim _{n \rightarrow \infty}\left\langle P_{+}(x, 0) P_{-}(y, 0)\right\rangle^{(n)},
$$

where

$$
P_{ \pm}(X)=\frac{1 \pm \sigma(X)}{2}, \quad X \in \mathbf{Z}^{d} \times \frac{1}{n} \mathbf{Z}
$$

We now apply Peierls' contour argument to the right-hand side to obtain

$$
\left\langle P_{+}(x, 0) P_{-}(y, 0)\right\rangle^{(n)} \leqq \sum_{\gamma \supset(x, 0)} e^{-E^{(n)}(\gamma)}
$$

where the sum is performed over all closed contours $\gamma$ in the dual lattice of $\mathbf{Z}^{d} \times \frac{1}{n} \mathbf{Z}$ 
enclosing the point $(x, 0)$. For the deterministic model with $h(x)=h$ for $x \in \mathbf{Z}^{d}$,

$$
E^{(n)}(\gamma)=\left|\gamma_{h}\right| 2 K_{n}+\left|\gamma_{v}\right| 2 \frac{J}{n}
$$

where $\gamma_{h}$ are the horizontal elements of $\gamma$ and $\gamma_{v}$ the vertical elements of $\gamma$. We now perform the sum over $\gamma$ by fixing $\left|\gamma_{h}\right|=L$, and an upper bound is obtained by summing over all possible numbers of vertical steps (with no immediate returns) after each horizontal step:

$$
\begin{aligned}
& \left\langle P_{+}(x, 0) P_{-}(y, 0)\right\rangle^{(n)} \times \sum_{L=2}^{\infty} L^{d}(2 d)^{L} e^{-2 K_{n} L} \times\left(\frac{2(2 d-1)}{1-e^{-2 J / n}}\right)^{L} \\
& =\sum_{L=2}^{\infty} L^{d}\left(\frac{4 d(d-1) \tanh \frac{h}{n}}{1-e^{-2 J / n}}\right)^{L} .
\end{aligned}
$$

Now

$$
\lim _{n \rightarrow \infty} \frac{\tanh \frac{h}{n}}{1-e^{-2 J / n}}=\frac{h}{2 J}
$$

and for $n>J, \frac{\tanh \frac{h}{n}}{1-e^{-2 J / n}}<\frac{h}{2 J}$ and therefore,

$$
\begin{aligned}
\left\langle P_{+}(x) P_{-}(y)\right\rangle & \leqq \sum_{L=2}^{\infty} L^{d}\left(\frac{4 d(2 d-1) h}{J}\right)^{L} \\
& \leqq\left(\frac{4 d(2 d-1) h}{J}\right)^{2} c_{d}\left(\frac{h}{J}\right) \quad \text { if } \quad\left(\frac{4 d(d-1) h}{J}\right)<1,
\end{aligned}
$$

where: $c_{d}(x)$ is monotonically increasing in $x$ for $x \geqq 0, c_{d}(0)=2 d$. Therefore for all $x, y \in \mathbf{Z}^{d},\left\langle\sigma_{3}(x) \sigma_{3}(y)\right\rangle \geqq a>0$ for some $a>0$, provided $\left(\frac{4 d(2 d-1) h}{J}\right)^{2} c_{d}<\frac{1}{2}$. The above discussion may be summarized as follows.

Theorem 5.1. Let $d \geqq 1$ and consider the d-dimensional deterministic model with $h(x) \equiv h$. Then there exists $h_{c}(J, d)>0$, monotonically increasing in $J$ with $\lim _{J \rightarrow \infty} h_{c}(J, d)=\infty$, such that if $h<h_{c}(J, d)$, there exists $a(h, J, d)>0$ with

$$
\left\langle\sigma_{3}(x) \sigma_{3}(y)\right\rangle \geqq a(h, J, d)
$$

for all $x, y \in \mathbf{Z}^{d}$.

Proof. From the above discussion $h_{c}(J, d) \geqq \bar{h}(J, d)$, where

$$
\left(\frac{4 d(2 d-1) \bar{h}}{J}\right) c_{d}\left(\frac{\bar{h}}{J}\right)=\frac{1}{2} .
$$

Monotonicity of $h_{c}(J, d)$ follows from Griffiths inequalities. 
Remark 5.2. If we consider the deterministic model restricted to a half space $\mathbf{Z}_{+}^{d}=\left\{\left(x_{1}, \ldots, x_{d}\right) \in \mathbf{Z}^{d}, x_{1} \geqq 0\right\}$ the same results with essentially the same proof hold true with $h_{c}^{+}(J, d)$ and $a^{+}(d, J h)$ substituting for the corresponding quantities. From Griffiths inequalities it follows that

$$
h_{c}^{+}(J, d)<h_{c}^{d}(J)
$$

To prove long range order for the random system we first introduce the independent site percolation model in $\mathbf{Z}^{d}$, where a site $x \in \mathbf{Z}^{d}$ is said to be occupied if $h(x) \leqq(1-\varepsilon) h_{c}^{+}(J, d)$, for $0<\varepsilon<1$. Therefore the probability of occupation of a site is

$$
p(J)=\mathbf{P}\left(h \leqq(1-\varepsilon) h_{c}^{+}(J, d)\right) .
$$

Now, from Theorem 5.1 and Remark $5.2 h_{c}^{+}(J, d) \rightarrow \infty$ as $J \rightarrow \infty$ and therefore there exists $J_{2}$ such that $p(J)>p_{c}^{(d)}$ for all $J>J_{2}$, where $p_{c}^{(d)}$ is the critical value for the $d$ dimensional site percolation problem. So, if $J>J_{2}$ with strictly positive P-probability there exists an infinite selfavoiding path $w$ of occupied sites starting at the origin:

$$
\begin{aligned}
& w:\{0,1,2, \ldots\} \rightarrow \mathbf{Z}^{d} \\
& i \rightarrow w_{i} ; w_{0}=0, \quad w_{i} \neq w_{j} \quad \text { if } i \neq j, \quad\left|w_{i+1}-w_{i}\right|=1 .
\end{aligned}
$$

We then consider the model in $\mathbf{Z}^{d}$ given by

$$
H_{w}=-J \sum_{i=0}^{\infty} \sigma_{3}\left(w_{i}\right) \sigma_{3}\left(w_{i+1}\right)-\sum_{x \in \mathbf{Z}^{d}} h(x) \sigma_{1}(x) .
$$

Notice that the points $x \in \mathbf{Z}^{d}, x \neq w_{i}$ for all $i$, are completely decoupled from the points in $w$. Therefore, the corresponding correlation functions are given by:

$$
\left\langle\sigma_{3}\left(w_{i}\right) \sigma_{3}\left(w_{j}\right)\right\rangle_{w}=\left\langle\sigma_{3}(i) \sigma_{3}(j)\right\rangle^{(1)}
$$

where the right-hand side is the two-point function of a one dimensional model in the half line with $h(i) \leqq(1-\varepsilon) h_{c}(J)$ for every $i \in \mathbf{Z}_{+}$. Therefore, from Theorem 5.1 and Remark 5.2

$$
\left\langle\sigma_{3}\left(w_{0}\right) \sigma_{3}\left(w_{i}\right)\right\rangle_{w} \geqq a>0 .
$$

From Griffiths inequalities it follows that

$$
\left\langle\sigma_{3}\left(w_{0}\right) \sigma_{3}\left(w_{j}\right)\right\rangle \geqq\left\langle\sigma_{3}\left(w_{0}\right) \sigma_{3}\left(w_{j}\right)\right\rangle_{w} \geqq a>0
$$

which implies:

$$
\varlimsup_{y \rightarrow \infty}\left\langle\sigma_{3}(0) \sigma_{3}(y)\right\rangle \geqq a>0 .
$$

Ergodicity then implies that, with probability one, there exists $z \in \mathbf{Z}^{d}$ such that

$$
\varlimsup_{|y| \rightarrow \infty}\left\langle\sigma_{3}(z) \sigma_{3}(y)\right\rangle \geqq a>0 .
$$

As in [9] we now use the Harris-FKG [13] inequality, whose validity is guaranteed by the path-space approximation, to get

$$
\left\langle\sigma_{3}(x) \sigma_{3}(y)\right\rangle \geqq\left\langle\sigma_{3}(x) \sigma_{3}(z)\right\rangle\left\langle\sigma_{3}(z) \sigma_{3}(y)\right\rangle .
$$


This implies that if $J>J_{2}$, with probability one

$$
\varlimsup_{|y| \rightarrow \infty}\left\langle\sigma_{3}(x) \sigma_{3}(y)\right\rangle>0
$$

for every $x \in \mathbf{Z}^{d}$, thus proving Theorem 1.2.

\section{References}

1. Ma, M., Halperin, B. I., Lee, P. A.: Phys. Rev. B34, 3136-3143 (1986)

2. Klein, A., Fernando Perez, J.: Localization in the ground state of the one-dimensional $x-y$ model with a random transverse field. Commun. Math. Phys. 128, 99-108 (1990)

3. Lieb, E. H., Schultz, T. D., Mattis, D. C.: Two soluble models of an antiferromagnetic chain. Ann. Phys. 16, 407-466 (1961)

4. de Gennes, P. G.: Solid State Commun. 1, 132 (1963)

5. Wang, Y. L., Cooper, B.: Phys. Rev. 172, 539 (1968)

6. Pfeuty, P.: The one-dimensional Ising model with a transverse field. Ann. Phys. 57, 79-90 (1970)

7. Ginibre, J.: Existence of phase transitions for quantum lattice systems. Commun. Math. Phys. 14, 205-234 (1969)

8. Driessler, W., Landau, L. J., Fernando Perez, J.: Estimates of critical lengths and critical temperatures for classical and quantum lattice systems. J. Stat. Phys. 20, 123-162 (1979)

9. Campanino, M., Klein, A.: Decay of two-point functions for $(d+1)$-dimensional Percolation, Ising and Potts Models with $d$-Dimensional Disorder. Commun. Math. Phys. 135, 483-497 (1991)

10. Klein, A., Landau, L. J.: Singular perturbations of positivity preserving semigroups via path space techniques. J. Funct. Anal. 20, 44-82 (1975)

11. Aizenman, M., Chayes, J. T., Chayes, L., Newman, C. M.: Discontinuity of the magnetization in one-dimensional $\frac{1}{|x-y|^{2}}$ Ising and Potts model. J. Stat-Phys. 50, 1-40 (1988)

12. Fisher, M.: Critical temperatures of anisotropic lattices II. General upper bounds. Phys. Rev. 162, 480 (1967)

13. Fortuin, C., Kasteleyn, P., Ginibre, J.: Correlation inequalities on some partially ordered sets. Commun. Math. Phys. 72, 89-103 (1971)

Communicated by T. Spencer

Note added in proof. Theorem 1.2 has been extended to $d=1$ by M. Aizenman and A. Klein if $\mathbf{E}\left(e^{\delta h(x)}\right)<\infty$ for some $\delta>0$. 
